\title{
Quantum Hall fractions for spinless Bosons
}

\author{
N. Regnault and Th. Jolicoeurf \\ Laboratoire Pierre Aigrain, ENS, Département de Physique, 24, rue Lhomond, 75005 Paris, France
}

\begin{abstract}
We study the Quantum Hall phases that appear in the fast rotation limit for Bose-Einstein condensates of spinless bosonic atoms. We use exact diagonalization in a spherical geometry to obtain low-lying states of a small number of bosons as a function of the angular momentum. This allows to understand or guess the physics at a given filling fraction $\nu$, ratio of the number of bosons to the number of vortices. This is also the filling factor of the lowest Landau level. In addition to the well-known Bose Laughlin state at $\nu=1 / 2$ we give evidence for the Jain principal sequence of incompressible states at $\nu=p /(p \pm 1)$ for a few values of $p$. There is a collective mode in these states whose phenomenology is in agreement with standard arguments coming e.g. from the composite fermion picture. At filling factor one, the potential Fermi sea of composite fermions is replaced by a paired state, the Moore-Read state. This is most clearly seen from the half-flux nature of elementary excitations. We find that the hierarchy picture does not extend up to the point of transition towards a vortex lattice. While we cannot conclude, we investigate the clustered Read-Rezayi states and show evidence for incompressible states at the expected ratio of flux vs number of Bose particles.
\end{abstract}

PACS numbers: 03.75Kk, 05.30.Jp, 73.43.Cd, 73.43.Lp

\section{INTRODUCTION}

Right from the beginning of the theory of the fractional Quantum Hall effect (FQHE) [1], it has been clear that the appearance of incompressible liquids is due to the interplay of repulsive interactions and the peculiar dynamics of the lowest-lying Landau level (LLL) but is not tied in any fundamental way to the statistics of the intervening particles. For example, in the hierarchy scheme described by Haldane in ref. [2] the quasiparticles are described by the Bose statistics and as a consequence they condense into Laughlin states with an even power of the relative separation. While theoretically important, this point has not received much attention till the advent of trapped ultra-cold atomic vapors that can be set in rotation [3, 田, 6, 6, (7). It is now feasible to gather a large number of bosonic atoms in a trap (magneto-optical or purely optical) and to manipulate many parameters in a controlled way. Notably one can create rotating Bose-Einstein condensates. They display the various phenomena expected for a rotating superfluid. When the velocity is large enough vortices are nucleated and eventually when their number grows they form the celebrated Abrikosov triangular lattice. This lattice can be considered as an elastic medium which has peculiar vibration modes. It has been pointed out in ref. [8] that for a special relation between the total angular momentum of the system and its number of particles, the ground state for bosons with a $\delta$ interaction is exactly a Laughlin wavefunction corresponding to a filling $\nu=1 / 2$ of the LLL when the system is in a two-dimensional (2D) regime. So, for large angular momentum the physics of the vortex lattice should be replaced by that of the FQHE for bosons [9, 10, 11]. If we consider a trap which is strongly confining along one direction so that the motion is effectively $2 \mathrm{D}$, then in the perpendicular plane particles will feel a (in general) harmonic restoring force. If the system is rotating there will be an additional centrifugal force counteracting the restoring force. If the rotation frequency equals the characteristic frequency of the harmonic trap, then we are left with only the Coriolis force which is formally equivalent to a magnetic field. The bosons will then be exactly in the conditions expected for the appearance of the FQHE. The extensive degeneracy of the one-particle problem, i.e. the degeneracy of the LLL will be lifted by interactions. For spinless bosons at ultracold temperatures, the scattering takes place only in the $s$ wave and so there is an effective local $\delta$ interaction between particles.

So far, the FQHE has only been observed experimentally for fermions with Coulomb interactions (including spin and layer index in bilayer structures). It is thus of prime importance to investigate the novelties that arise in the Bose setting. It is natural to expect a whole hierarchy of incompressible liquids with the accompanying quasiparticles. The fractional charge means here excess or missing density. In addition to the Laughlin state at $\nu=1 / 2$ which is exact, the quasiholes created from this parent liquid are also exact zero-energy eigenstates of the Hamiltonian of this system. It has been suggested that the fractional statistics of these entities may be experimentally measured by laser manipulations [12]. There is evidence from exact diagonalizations of systems with a small number of particles for

*Electronic address: Nicolas.Regnault@lpa.ens.fr, Thierry.Jolicoeur@lpa.ens.fr 
a prominent sequence of fractions $\nu=n /(n \pm 1)$, akin to the so-called principal sequence $\nu=n /(2 n \pm 1)$ observed in fermion systems. While this is closely related to the physics of the fermion systems, there is also evidence [11] for more exotic states intervening in the filling range between $\nu \approx 1$ and the melting transition of the vortex lattice which may take place for $\nu \approx 6$. For the filling $\nu=1$, it has been suggested that the ground state is closely related to the so-called Pfaffian state of Moore and Read [13 proposed to describe fermions at $\nu=5 / 2$. One can describe this Pfaffian state by pairing of fictitious composite fermions. The pairing has two signatures that can be seen in numerical experiments : there is a special shift in the relation between the flux and the number of particles in the spherical geometry and also quasiparticles are created in pairs by adding one extra flux quantum onto the fiducial ground state. For fillings $\nu>1$, there is some evidence from exact diagonalizations in the torus geometry that the incompressibility occurs through the Read-Rezayi states 14. These states are generalizations of the Pfaffian and involve the clustering of $k$ composite particles at filling $\nu=k / 2$ in their simplest version. They seem to fill the gap between the Pfaffian state at $\nu=1$ and the phase transition to the vortex lattice. The critical filling for this transition has been evaluated from exact diagonalization : the ordering is identified by the appearance in the spectrum of low-lying states with quantum numbers appropriate to the breaking of translational invariance in the torus geometry [11]. A wealth of new quantum Hall states is also predicted for bosons with spin 15, 16. Some of them are generalizations of the clustered Read-Rezayi states. For spinless bosons, there is so far no rigorous proof that exotic paired states are realized in the realistic case of pure s-wave scattering. However if one gets close to a so-called Feshbach resonance the resulting mixture of atoms and molecules may admit such exotic states as rigorous ground states [17].

In this paper, we give the detailed results of a study of the FQHE for spinless bosons by exact diagonalization mainly in the spherical geometry. By studying the low-lying levels of small systems, we identify candidates for series of fractions displaying incompressibility. We give evidence for the existence of a Pfaffian-like ground state at $\nu=1$. We have also studied larger fillings and have identified potential candidates for the Read-Rezayi states that have so far only been observed in the torus geometry. We examine ground state degeneracy as well as the peculiar charged excitations. Some of our results were announced in a Letter [18].

In section II, we give some details of the formalism used to treat the Bose problem on the disk and on the sphere. In section III, we give evidence for the principal sequence of fractions. Section IV is devoted to the filling $\nu=1$ and its relation to the Pfaffian. Section V contains the results about fillings larger than one. Finally section VI contains our conclusions.

\section{FORMALISM : FROM THE DISK TO THE SPHERE}

In the rotating frame, the Hamiltonian describing $\mathrm{N}$ interacting bosons of mass $m$ is given by :

$$
\mathcal{H}=\sum_{i=1}^{N} \frac{1}{2 m}\left(\mathbf{p}_{i}-m \omega \hat{\mathbf{z}} \times \mathbf{r}_{i}\right)^{2}+\frac{1}{2} m\left(\omega_{0}^{2}-\omega^{2}\right)\left(x_{i}^{2}+y_{i}^{2}\right)+\frac{1}{2} m \omega_{z}^{2} z_{i}^{2}+\sum_{i<j}^{N} V\left(\mathbf{r}_{i}-\mathbf{r}_{j}\right) .
$$

Here, the angular velocity is $\omega$ along the $\hat{\mathbf{z}}$ axis, the $x y$ trap frequency is $\omega_{0}$ and the characteristic frequency of the trapping $z$ potential is $\omega_{z}$. We have a $2 \mathrm{D}$ regime when the confinement along $z$ is strong enough to freeze the motion in its ground state. When we tune the rotation close to criticality $\omega \approx \omega_{0}$, we are dealing with the problem of bosons in an effective field $\mathbf{B}=(2 m \omega / e) \hat{\mathbf{z}}$. There is a corresponding magnetic length $\ell=\sqrt{\hbar /(2 m \omega)}$. The one-body problem displays the Landau levels with a degeneracy given by $1 / 2 \pi \ell^{2}$ state per unit area. In the language of the quantum Hall effect, it is convenient to define the filling factor $\nu=n \times 2 \pi \ell^{2}$ where $n$ is the areal density of bosons. In the language of rotating traps the filling factor $\nu$ is the ratio of the number of bosons to the average number of vortices. For large number of particles and vortices $\nu \approx N^{2} / 2 L_{z}$ where $L_{z}$ is the angular momentum along the $\hat{\mathbf{z}}$ axis. When we are exactly at the point $\omega=\omega_{0}$, the system has a degeneracy which is lifted only by the interactions. In the case of ultracold bosons, the scattering between atoms takes place only in the $s$-wave. As a consequence, it is an excellent approximation to describe interactions by an instantaneous potential which is a delta function :

$$
V(\mathbf{r})=\frac{4 \pi \hbar^{2} a_{s}}{m} \delta^{(3)}(\mathbf{r})
$$

where $a_{s}$ is the $s$-wave scattering length. We consider the repulsive case $a_{s}>0$. If we are in a $2 \mathrm{D}$ regime, the wavefunction along $\mathrm{z}$ axis will be the ground state of the harmonic oscillator and thus the effective potential felt in the plane is :

$$
V^{(2 D)}(\mathbf{r}) \equiv \sqrt{32 \pi} \hbar \omega \frac{a_{s} \ell^{2}}{\ell_{z}} \delta^{(2)}(\mathbf{r})
$$


In this equation, $\ell_{z}$ is the characteristic length of the z-axis oscillator : $\ell_{z}=\sqrt{\hbar / m \omega_{z}}$. Since the kinetic energy is totally quenched, all characteristic energies will be given by pure numbers times the energy scale given by the dimensionfull coefficient appearing in $V^{(2 D)}(\mathbf{r}): g=\sqrt{32 \pi} \hbar \omega\left(a_{s} / \ell_{z}\right)$. The separation of the successive Landau levels is given by $\hbar \omega_{c}=2 \hbar \omega$. Comparing this cyclotron energy to the scale $g$ set by interactions, we see that Landau level mixing will be small if $a_{s} / \ell_{z}$ is small enough.

The Hamiltonian in Eq.(1) is that of charged particles in the symmetric gauge with a vector potential $\mathbf{A}(\mathbf{r})=\frac{1}{2} \mathbf{B} \times \mathbf{r}$. We will from now on concentrate on the lowest Landau level only. The eigenstates of the one-body problem are then simply given by functions of the complex coordinate $z=x+i y$ :

$$
\phi_{m}(z)=\frac{1}{\sqrt{\ell \sqrt{\pi}}} z^{m} \mathrm{e}^{-|z|^{2} / 4 \ell^{2}} .
$$

The positive integer $m$ is the angular momentum of the state : $L_{z}=m \hbar$. Due to the very special dynamics of the LLL, it is easy to solve the two-body problem for an arbitrary interaction between particles. The eigenstate of the relative particle is taken to be of the form Eq.(4) and then the associated eigenenergy is $\hbar \omega_{c}+V_{m}$ where $V_{m}=\left\langle\phi_{m}|V| \phi_{m}\right\rangle$. The coefficient $V_{m}$ is the interaction energy of two particles with relative angular momentum $m$. These coefficients called pseudopotentials are a convenient way to parameterize interactions [19]. In the case of the pure delta interaction, only $V_{0}$ is different from zero. In the case of the Coulomb interaction all pseudopotentials are non zero and only the ones with even $m$ contribute to the interaction between bosons.

In order to be entirely in the LLL, an arbitrary many-body state $\Psi$ should be of the following from :

$$
\Psi\left(x_{1}, y_{1}, \ldots, x_{N}, y_{N}\right)=P\left(z_{1}, \ldots, z_{N}\right) \prod_{k=1}^{N} \mathrm{e}^{-\left|z_{k}\right|^{2} / 4 \ell^{2}}
$$

where $P$ should be an analytic function of each of the $z_{i}$ complex coordinates. The Bose statistics requires $P$ to be symmetric.

The many-body problem so defined is finite-dimensional even in the unbounded plane once we fix the total angular momentum $L_{z}^{\text {tot }}$ since all the states in Eq.(4) have positive angular momentum. So for a small number of bosons one may diagonalize exactly the Hamiltonian in the disk geometry in sectors of fixed $L_{z}$. The ground state energy is then a decreasing function of $L_{z}^{\text {tot }}$ since the particles interact only for zero relative angular momentum. Zero ground state energy is reached for $L_{z}=N(N-1)$ by the celebrated Laughlin-Jastrow wavefunction :

$$
\Psi_{L J}^{(2)}=\prod_{i<j}\left(z_{i}-z_{j}\right)^{2} \prod_{k=1}^{N} \mathrm{e}^{-\left|z_{k}\right|^{2} / 4 \ell^{2}} .
$$

It is only starting from this value of $L_{z}$ that all particles can completely avoid each other. For the special condition $L_{z}=N(N-1)$ this is in fact the only zero-energy eigenstate. For larger values of the angular momentum, there are more and more zero-energy states obtained by multiplying $\Psi_{L J}^{(2)}$ by an arbitrary symmetric polynomial $Q$ of the $z_{i}$ coordinates :

$$
\Psi=Q\left(z_{1}, \ldots, z_{N}\right) \times \Psi_{L J}^{(2)}
$$

It is well known from the theory of the fermionic FQHE that when the degree of $Q$ is small enough, the states Eq.(7) describe edge excitations of the Laughlin droplet [20]. When the degree of the polynomial is of order $N$, one generates quasiholes. the polynomial

$$
Q\left(z_{1}, \ldots, z_{N}\right)=\prod_{i}\left(z_{i}-Z_{0}\right)
$$

will generate a quasihole located at $Z_{0}$. The delta function interaction for which the Laughlin wavefunction is an exact ground state has also the properties that the quasiholes are exact, zero-energy eigenstates. This however does not extend to the quasielectrons as we will see below. When $Q$ has a degree of order $N^{2}$, it will finally change the filling factor of the LLL.

A sample spectrum is displayed in Fig.(1) for $\mathrm{N}=5$ bosons. Each state at the left boundary of the spectrum, i.e. the one with smallest $L_{z}^{\text {tot }}$ at fixed energy, generates a family of daughter states with increasing angular momentum. For zero energy, the family is the one we described above in Eq.(7). It is clear that when performing exact diagonalization in a sector with fixed $L_{z}$ we have to cope with all the descendants of the states with smaller angular momentum 21, 22, 23]. A more appealing scheme for such numerical studies is to use the spherical geometry in which levels can be 


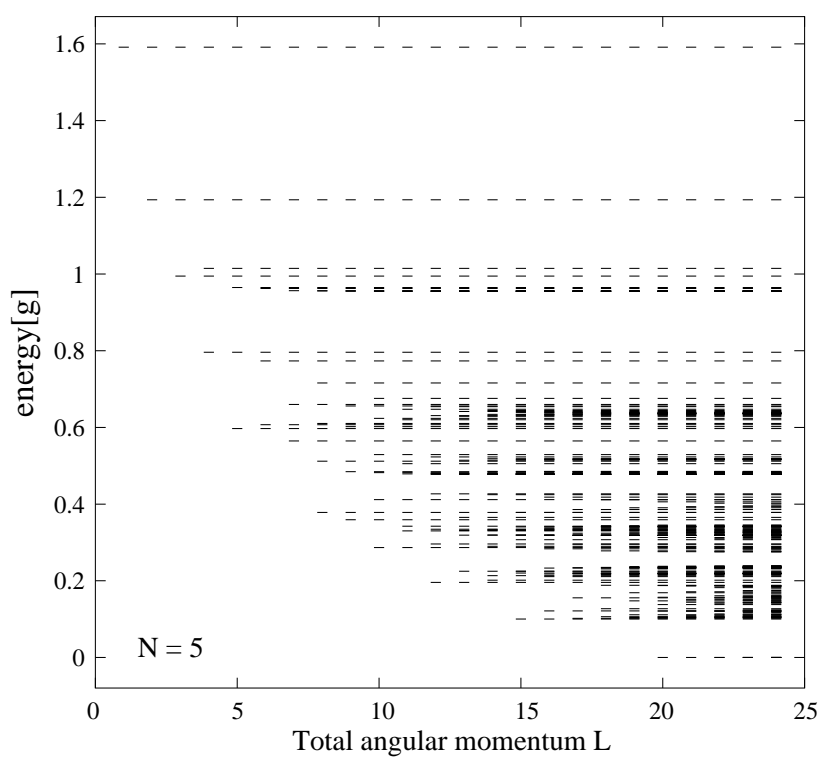

Figure 1: Energy spectrum for $\mathrm{N}=5$ bosons. Energies are in units of $g$ and the horizontal axis is total angular momentum. The Laughlin state appears at zero energy for $\mathrm{L}=20$.

classified by the full rotation group. It was shown by Haldane and Rezayi $[19$ that the essential phenomena of the FQHE can be understood from such calculations even for a relatively small number of fermions.

If the bosons reside on a sphere then we need a magnetic monopole at the center of the sphere in order to produce a uniform magnetic field on its surface. If $R$ is the radius of the sphere, then Dirac quantization condition requires that the total flux $4 \pi R^{2} B$ must be an integral multiple $2 S$ of the flux quantum $h / e$. Thus the magnetic field is proportional to $S: B=\hbar S / e R^{2}$. The one-body problem can then be solved in polar coordinates [24, 25] : the LLL has degeneracy $2 S+1$ and is spanned by the (unnormalized) basis functions :

$$
u^{S+M} v^{S-M}, \text { where } M=-S, \ldots,+S,
$$

and $u$ and $v$ are the spinors in spherical coordinates :

$$
u=\cos (\theta / 2) \mathrm{e}^{i \varphi / 2}, v=\sin (\theta / 2) \mathrm{e}^{-i \varphi / 2} .
$$

The sphere and the unbounded disk can be related by a stereographic projection 26] that allows to convert explicit wavefunctions from one geometry to the other. The previous Laughlin wavefunction Eq.(6) becomes on the sphere :

$$
\Psi^{(2)}=\prod_{i<j}\left(u_{i} v_{j}-u_{j} v_{i}\right)^{2}
$$

In order to reside entirely in the LLL, the total degree in $u$ and $v$ of an arbitrary wavefunction for $\mathrm{N}$ particles should be $2 N S$. The above function $\Psi^{(2)}$ has total degree $2 N(N-1)$ so it will be an acceptable state only for the precise matching between flux and the number of particles $2 S=2(N-1)$. When both $N$ and $2 S$ are large, this is what we expect from the filling factor $\nu=1 / 2$ of the function $\Psi^{(2)}$. However for all finite values there is a nontrivial shift between finite system matching condition and its thermodynamic limit. This shift has a topological origin and can be used to discriminate between candidates for incompressible states. It will be useful in our study of the Pfaffian state described in section IV.

On the sphere, total angular momentum is conserved and the many-body eigenstates can be classified by their $L_{t o t}$ value. An incompressible quantum Hall fluid which breaks no symmetry is expected to appear as an isolated $L=0$ singlet separated by a sizable gap from the excited states. By comparison, broken symmetry states appear as a set of quasi-degenerate states. The gap above the isolated singlet is the hallmark of incompressibility, the most salient feature of FQHE states. We have performed a systematic search for incompressible states in the $(N, 2 S)$ plane for spinless bosons with the pure delta interaction. We have used the Lánczos algorithm to obtain some of the low-lying eigenvalues. Since incompressible states do not break any symmetry, we expect that the corresponding spectrum has a singlet $L=0$ ground state clearly separated by a gap from higher lying states. Of course this gap should remain 
nonzero at the thermodynamic limit, a fact that can be established only by careful finite-size scaling. In Fig.(2) we display all states with a singlet ground state that we have been able to study numerically. Some of these states are candidates for an incompressible state. However this is not the case for all the points in Fig.(2). When the number of bosons per state becomes quite large, we find spectra that look like uncorrelated rotors with no sign of gapped modes. This is the case for $2 \mathrm{~S}=2,3$ at all fillings, i.e. all numbers of bosons. Of course, the criteria mentioned above do not allow for an unambiguous selection. One needs to find series of states converging towards a thermodynamic limit. They should appear along lines of fixed slope, i.e. filling factor, in the $(N, 2 S)$ plane. In Fig.(2) we have plotted the lines corresponding to the FQHE states $\nu=1 / 2,2 / 3$ as well as the Moore-Read state for $\nu=1$ (to be discussed below) and also the fraction $\nu=2$ from the unpaired hierarchy (this last one does not converge to the thermodynamic limit, see sect. V).

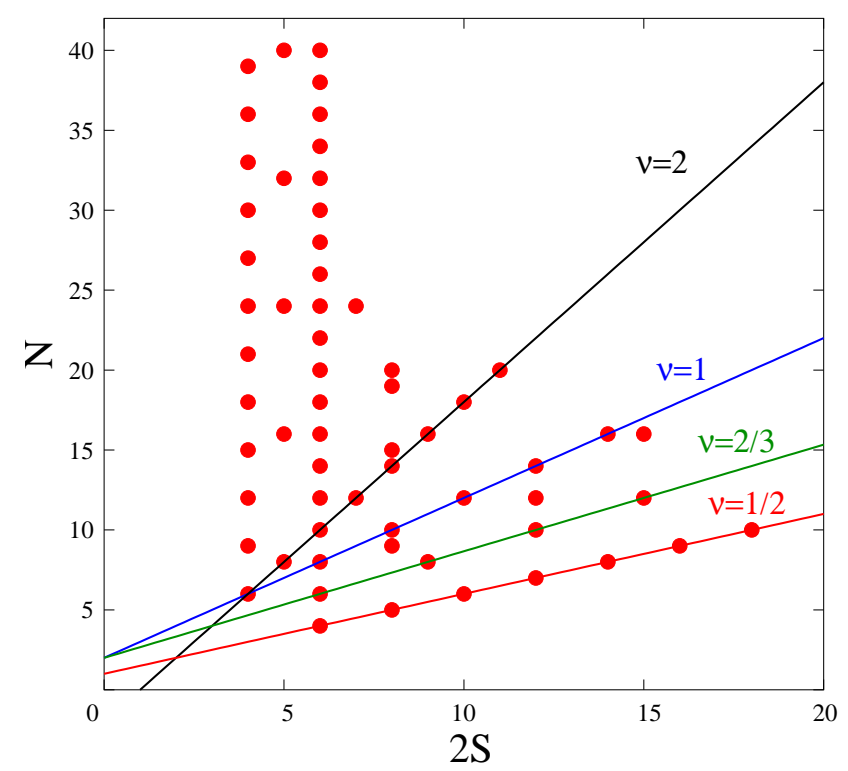

Figure 2: Candidates for incompressible states in the plane (number of particles, flux). Dots indicate a $L=0$ ground state. The lines show the flux-number of particles relationships of some FQHE states.

\section{THE PRINCIPAL SEQUENCE}

\section{A. The $\nu=1 / 2$ Laughlin state}

For $2 S=2(N-1)$ we obtain the typical spectra from the prototypical incompressible Laughlin state : see Fig.(3). The Laughlin state itself is the $L=0$ ground state. It is separated by a clear gap from a set of states that are themselves separated from the quasi-continuum of excited states. The well-defined branch extend from $L=2$ up to $L=N$ and is a collective excitation branch [27]. This is close to the case of fermions with Coulomb interactions but there is one important difference : here this branch has no clear minimum before it ends. In the case of fermions, there is a minimum, the magnetoroton, that is attributed to the incipient instability towards formation of a Wigner-crystal with a well-defined lattice spacing, hence defining a typical wavevector. In the case of a short-range interaction, we do not expect any instability of this kind and hence no specific wavevector should be singled out in the dispersion relation (on the sphere wavevector correspond to angular momentum by $k R=L$ ). We have evaluated [18] the gap between the ground state and the collective mode by performing finite-size scaling up to $\mathrm{N}=10$ bosons : the result is $0.95(5) \mathrm{g}$. This estimate does not vary much when we use the extremal point $L=N$ of the collective mode or any point in the flat part.

If we add one quantum of flux, then we create a single quasihole with $L=N / 2$ which is gapless (see section II). If we remove one flux quantum, the quasielectron with also $L=N / 2$ has a gap and no exact result is known for its wavefunction. Similarly we have computed the gap for quasielectrons for up to N=12 bosons and extrapolated to the thermodynamic limit. The result is the same gap as above for the collective mode. This is what we expect for a quasihole-quasielectron bound state. 


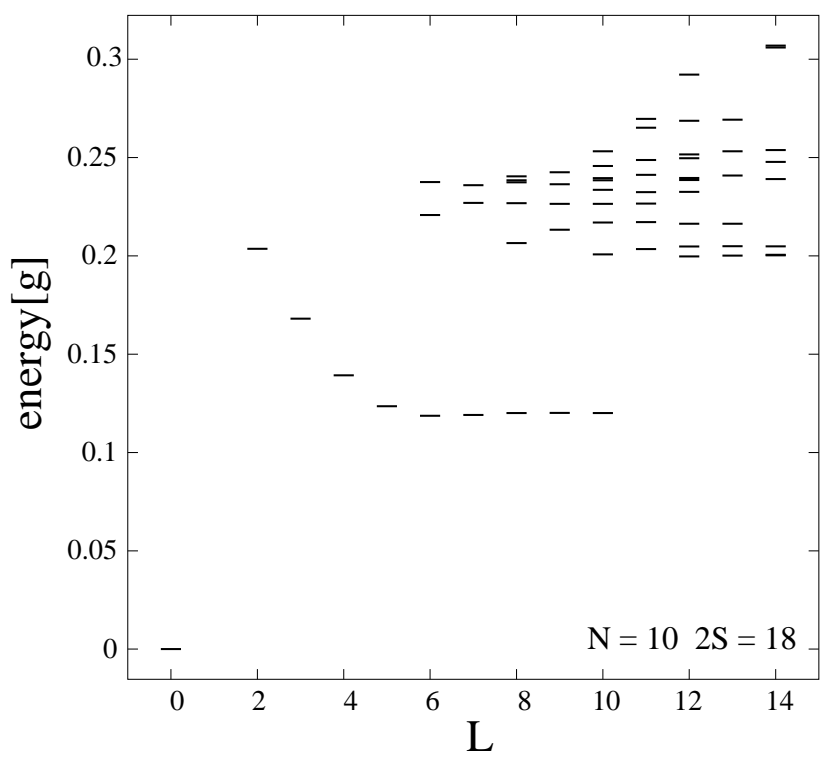

Figure 3: Energy spectrum for $\mathrm{N}=10$ bosons at $2 \mathrm{~S}=18$ corresponding to the filling $\nu=1 / 2$. The Laughlin state is the $L=0$ ground state at zero energy. There is a clear collective mode branch that extends up to $L=N=10$. The upper part of the spectrum has been truncated for clarity but extends continuously. Energies are in units of $g$ and the horizontal axis is total angular momentum.

\section{B. The principal sequence}

Starting from the state $\nu=1 / 2$ quasiparticle condensation into successive Laughlin states generate a whole hierarchy of incompressible states. In the case of fermions the most prominent series are given by $\nu=n /(2 n \pm 1)$. This socalled principal sequence [28] can be derived from the composite fermion scheme [29] which is one of the variants of the hierarchy theory. This can be easily translated for bosons. Attaching a flux tube with one quantum of statistical flux transmute the boson into a (composite) fermion. In a mean-field picture the fictitious magnetic field compensates partially the external applied field and the composite fermions then populate Landau levels leading to incompressibility when an integer $p$ of these levels are filled. With one flux tube per fermion the filling of the original bosons is $\nu=p /(p \pm 1)$. On the sphere the same reasoning leads to the relations :

$$
2 S=\frac{p \pm 1}{p} N \mp p-1 \quad \text { for } \quad \nu=\frac{p}{p \pm 1} .
$$

From the accessible sizes, we have evidence for $\nu=2 / 3$ realized for $\mathrm{N}=4,6,8,10,12$. The lower part of the spectrum for $\mathrm{N}=12,2 \mathrm{~S}=15$ is displayed in Fig.(位). Since the filling is now higher than $1 / 2$ it is no longer possible that all bosons avoid interactions, hence the ground state no longer has zero energy. Above the isolated singlet ground state there is evidence for a gapped collective mode which is not as clearly resolved as in the case of the strongest fraction $\nu=1 / 2$. If we define the gap as the energy difference between the ground state and the first excited state irrespective of its angular momentum then an extrapolation to the thermodynamic limit in $1 / \mathrm{N}$ leads to a nonzero value of $0.05 \mathrm{~g}$. In the composite fermion scheme it is simple to compute the extend of the collective mode. The lowest energy neutral excitation can be obtained by promoting a composite fermion from the highest occupied $p^{\text {th }}$ Landau level to the first empty $p+1^{\text {th }}$ level. Since the angular momenta are then $L_{C F}=(N / 2 p)+(p / 2)$ and $L_{h}=(N / 2 p)+(p / 2)-1$ respectively for the fermion and the hole, it follows that the particle-hole state has maximal angular momentum $L_{\max }=(N / p)+p-1$. This is consistent with what we observe in Fig.(4). For the Laughlin state with $\mathrm{p}=1$, one has $L=N$ as the maximum value which is the same result as considering the collective mode as a quasihole-quasielectron pair. For all the hierarchical descendants, we find the general trend that the collective mode has apparently a downward curvature, as is also seen in the case of fermions with Coulomb interactions.

For the fraction $\nu=2 / 3$ we have also studied the charged excitations. From the composite fermion scheme we obtain that a single quasihole (resp. quasielectron) is created by removal (resp. addition) of a composite fermion, leading to the following flux matching condition :

$$
\text { quasiholes : } 2 S=\frac{p \pm 1}{p} N \mp p-1+\frac{1}{p} \quad \text { for } \quad \nu=\frac{p}{p \pm 1},
$$




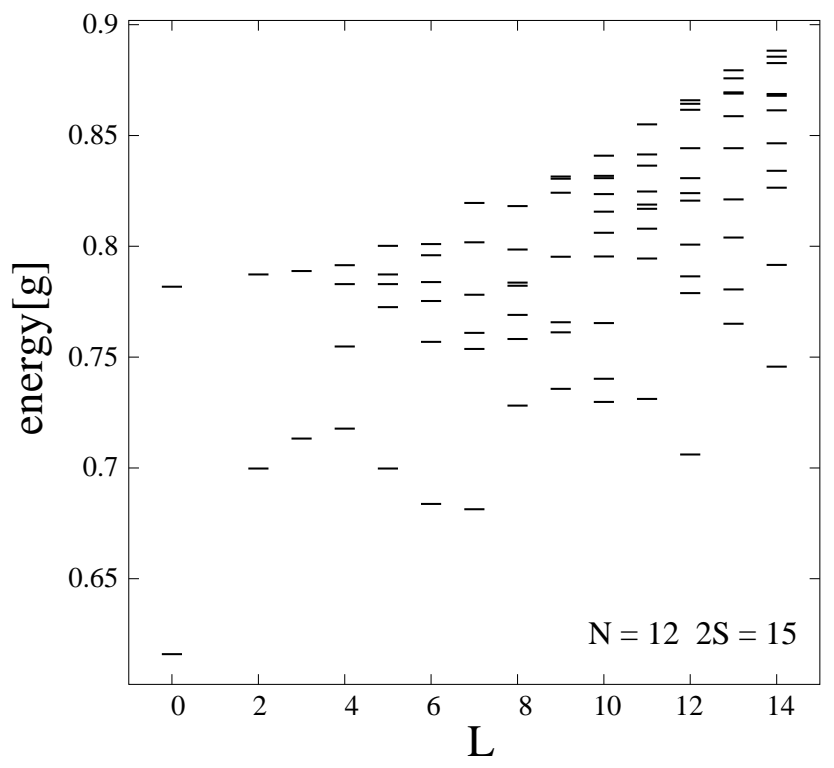

Figure 4: Energy spectrum for $\mathrm{N}=12$ bosons at $2 \mathrm{~S}=15$ corresponding to the filling $\nu=2 / 3$. Above the $L=0$ ground state there is a collective mode branch that extends up to $L=7$.

$$
\text { quasielectrons : } 2 S=\frac{p \pm 1}{p} N \mp p-1-\frac{1}{p} \quad \text { for } \quad \nu=\frac{p}{p \pm 1} .
$$

Due to the factor $1 / p$ it is not possible to create a quasiparticle without changing the number of bosons (except for the primary Laughlin state $\nu=1 / 2$ ). As a consequence when computing the gap to charged excitations one has to extrapolate the ground state energy of the parent fluid to a number of bosons at which it is not realized. For $\nu=2 / 3$ the gap estimate from charged excitations converges to a value close to the gap of the neutral excitations $0.05(1) \mathrm{g}$.

For the incompressible state at $\nu=3 / 4$ we have three candidates at $N=4,8,12$. They all consistently display a gap and the first branch of excited states also obeys the rule $L_{\max }=(N / p)+p-1$. There are too few points then to estimate the gap. Similarly we find two consistent candidates for $\nu=4 / 5$ at $\mathrm{N}=8$ and 12 . The same data set also points to the existence of at least the fractions $4 / 3$ and $5 / 4$. Indeed, there is aliasing [30] of the the (N,2S) points : a point with a given value of $\mathrm{N}$ and $2 \mathrm{~S}$ at fraction $\nu=p /(p+1)$ also satisfies condition $(12)$ for $\nu=p^{\prime} /\left(p^{\prime}-1\right)$ if $p p^{\prime}=N$. We thus find evidence for $\nu=4 / 3$ from $\mathrm{N}=8,12,16$ and $\nu=5 / 4$ for $\mathrm{N}=5,10$.

When the filling is less than $1 / 2$,zero-energy states proliferate and all other fractions are embedded in the angular momentum descendants of the Laughlin state. This is a peculiarity of the delta-function interaction and disappears if we change the interaction pseudopotential. If we add a $\mathrm{V}_{2}$ term then the most prominent new fraction is $\nu=2 / 5$. This fraction does not belong to the Jain principal sequence of states but appears if we consider the Haldane bosonic construction of the hierarchy : quasiholes on top of the $\nu=1 / 2$ state can themselves condense into a $\nu^{\prime}=1 / 2$ fluid leading to a $2 / 5$ state (while it is quasielectron condensation at $\nu^{\prime}=1 / 2$ that leads to the $2 / 3$ state).

\section{THE $\nu=1$ STATE}

If we consider the composite fermion picture, a striking prediction is that the effective magnetic field may be zero for certain filling factor. For fermions this happens when $\nu=1 / 2$ which is the limiting point of the Jain sequence $n /(2 n \pm 1)$ for $n \rightarrow \infty$. In the case of the Coulomb interaction there is evidence that some kind of Fermi sea of composite fermions is present. This comes from studies of Chern-Simons theory 31 and also from numerical studies [32 of spherical systems. Similarly for bosons, the effective magnetic flux vanishes for $\nu=1$ the limiting point of the sequence $n /(n \pm 1)$ for $n \rightarrow \infty$. So some kind of renormalized Fermi liquid is a likely candidate for the ground state at this special filling. It has been also pointed out first for the Fermi case that the effective Fermi sea may be unstable with respect to pairing of composite fermions. An appealing wavefunction describing in a BCS manner the pairing of the composite fermions is the so-called Pfaffian wavefunction [13, 33, 34. On the sphere it can be written :

$$
\Psi=\operatorname{Pf}\left\{\frac{1}{u_{i} v_{j}-u_{j} v_{i}}\right\} \prod_{i<j}\left(u_{i} v_{j}-u_{j} v_{i}\right)^{q} .
$$


The symbol Pf stands for the Pfaffian and $N$ should be even. Given an antisymmetric $N \times N$ matrix $A_{i j}$ it is defined by [35]:

$$
\operatorname{Pf}\left\{A_{i j}\right\}=\sum_{\sigma} \epsilon_{\sigma} A_{\sigma(1) \sigma(2)} \ldots A_{\sigma(N-1) \sigma(N)}
$$

where $\sigma$ are permutations of the index with $N$ values. For $q=1$ the wavefunction Eq.(15) describes bosons at filling factor $\nu=1$ while for $q=2$ it describes fermions at $\nu=1 / 2$. The filling factor is $\nu=1 / q$ and this wavefunction will fit on the sphere if we have :

$$
2 S=q(N-1)-1
$$

The wavefunction in Eq.(15) no longer vanishes when two particles are at the same point because of the denominator. It has thus no zero energy. However it still vanishes when three particles coincides. Using this property one can construct a special Hamiltonian for which it is the exact ground state [13]. Along a similar line, It has been also noted 17 that a gas of atoms and molecules in the condition of a Feshbach resonance may have also the Pfaffian as an exact ground state.

It has been proposed that the Pfaffian state describes the elusive $\nu=5 / 2$ incompressible state observed in twodimensional electron gases [36, 37]. One has to treat the LLL as inert and then the lower spin branch of the first excited Landau level which is at effective filling 1/2 may prefer the Pfaffian paired state. There is some evidence that this happens from numerical results on the sphere but this requires to tweak somewhat the pseudopotential between electrons. In the Bose $\nu=1$ case, studies in the torus geometry are consistent with the Pfaffian state being the ground state 11]. There were also early hints of this state 38 . We observe in the spherical geometry a strong series of incompressible candidates satisfying $2 S=N-2$ for $N=4,6,8,10,12,14,16$. Some of them are displayed in Fig.(5a,b,c). They have an isolated singlet ground state and a well-defined branch of neutral excitations made of states separated by $\Delta L=1$. The gap extrapolates to a nonzero value $18 \approx 0.1 \mathrm{~g}$. Even we use the less favorable fit, a quadratic fit starting from the smallest size so including the maximum amount of downward curvature in a $1 / \mathrm{N}$ plot, then the extrapolated value is still nonzero. There are also neutral fermionic excitations above the Pfaffian ground state. In the case of the bosonic problem, this fermion is a composite fermion being one bare boson with one additional flux tube [39]. From a paired state we obtain such a state by adding one boson and adding also one extra flux quantum. Since the paired state lies on the trajectory $2 S=N-2$ we are still on this line in the (N, 2S) plane but now with $\mathrm{N}$ odd. Consistently, we observe a strong even-odd effect on the Pfaffian line. Typical spectra for odd number of particles are given in Figs.(6a,b,c). They are gapless with a "hanging chain" shape of states separated by $\Delta L=1$ as observed in corresponding fermion systems [33].

An intriguing feature of the Pfaffian physics is the occurrence of unconventional excitations endowed with nonAbelian statistics [13]. This is especially interesting in the case of the Bose systems in magneto-optical traps since one may eventually perform laser manipulations of quasiparticles as suggested by Paredes et al. [12] to observe the non-Abelian mixing of quasiparticles induced by a real-space permutation of the positions. These excitations are created in pairs by addition or removal of a single quantum of flux from the magic condition Eq.(17). The low-lying states should form then a two-particle branch with a $\Delta L=2$ interval rule as expected for indistinguishable particles.
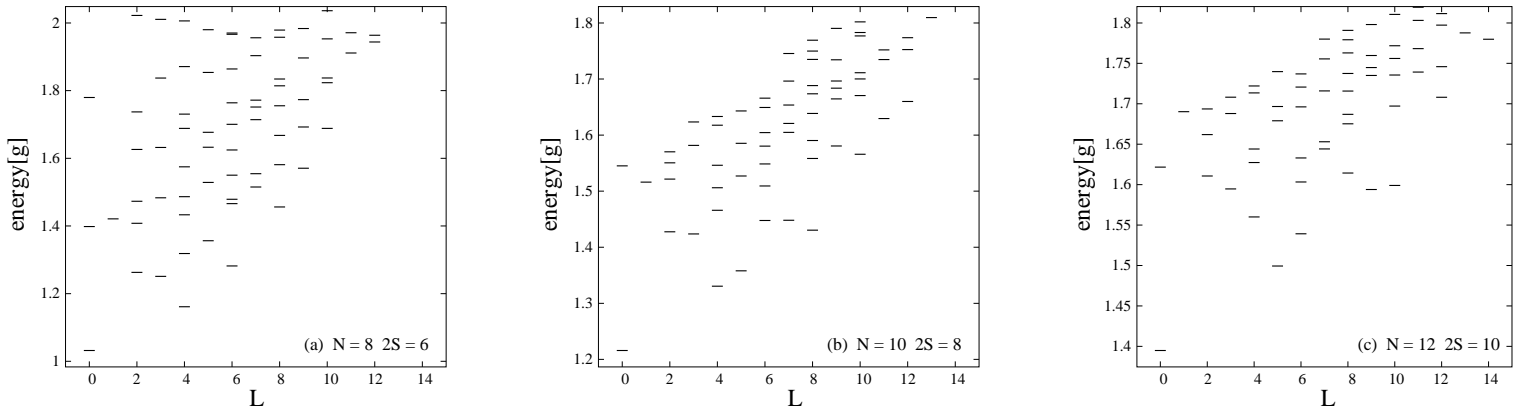

Figure 5: Incompressible states related to the Pfaffian when the number of particles is even : (a) $\mathrm{N}=8,(\mathrm{~b}) \mathrm{N}=10,(\mathrm{c}) \mathrm{N}=12$.

We have also studied the stability of the Pfaffian state by varying the interaction potential. This is done by adding more pseudopotentials to the pure hard-core $\mathrm{V}_{0}$ interaction. We use a Hamiltonian of the form $H_{0}+\lambda H_{1}$ where $H_{0}$ represents the pseudopotential $\mathrm{V}_{0}$ only and $H_{1}$ stands for all the even pseudopotentials $\mathrm{V}_{m}$ of the Coulomb interaction for $m \geq 2$. The full Coulomb interaction is restored for $\lambda=1$. This special point was studied in ref.(40]). For a $\mathrm{N}=6$ boson system on the sphere they found evidence for incompressibility when $2 S=4$, as expected for the Pfaffian 

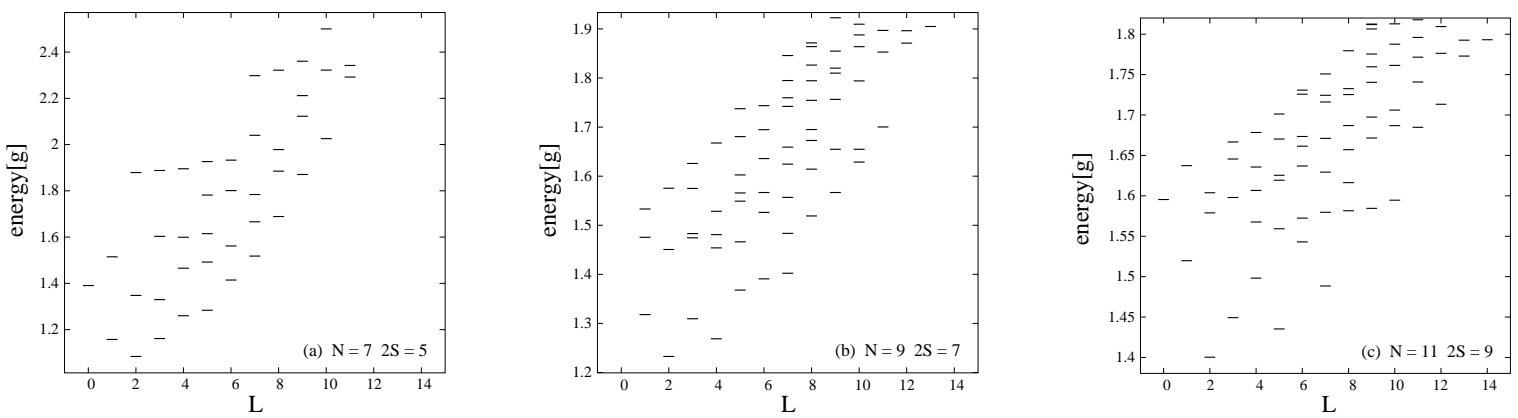

Figure 6: States with one pair-breaking excitation when the number of particles is odd : (a) $\mathrm{N}=7$, (b) $\mathrm{N}=9$, (c) $\mathrm{N}=11$.

state. We have computed the gap above the Pfaffian in the range $0 \leq \lambda \leq 1$. We find that the finite size law of the gap barely changes as we go to a long-range potential. In all cases the extrapolation is consistent with a nonzero gap. So it is likely that this paired state is robust. When $\lambda \neq 0$, the states for $\nu \leq 1 / 2$ are no longer zero-energy states and we find that the most prominent fraction that emerges is $\nu=2 / 5$.

\section{INCOMPRESSIBILITY AT $\nu>1$}

When the filling factor is large then it is likely that there will be a quantum phase transition towards the Abrikosov lattice of vortices already observed in trapped gases. Initial estimates 11] from exact diagonalizations in the torus geometry point to a critical filling $\nu_{c} \approx 6$ for the transition. A study using a Lindemann melting criterion for the vortex lattice also gives a similar result [41]: $\nu_{c} \approx 8$. It is thus of interest to understand the special region for fillings larger than one. There are hierarchy states belonging to the principal sequence : this is the case for $\nu=3 / 2$ and $\nu=2$ the termination point of the $p /(p-1)$ series of states. For $\nu=3 / 2$ we have only three candidates available $\mathrm{N}=6,9,12$ and $\mathrm{N}=12$ is aliased with the Pfaffian. While the corresponding spectra look incompressible, it is not possible to draw any firm conclusion concerning their survival at the thermodynamic limit. For $\nu=2$ on the contrary, the values $\mathrm{N}=6,8,10,12,14,16,18$ can be studied. In this series the neutral gaps as a function of $1 / \mathrm{N}$ are displayed in Fig.(7). There is no tendency to convergence. If we try to investigate charged excitations on top of the $\nu=2$ state, then we find only compressible states but no clear quasiparticles.

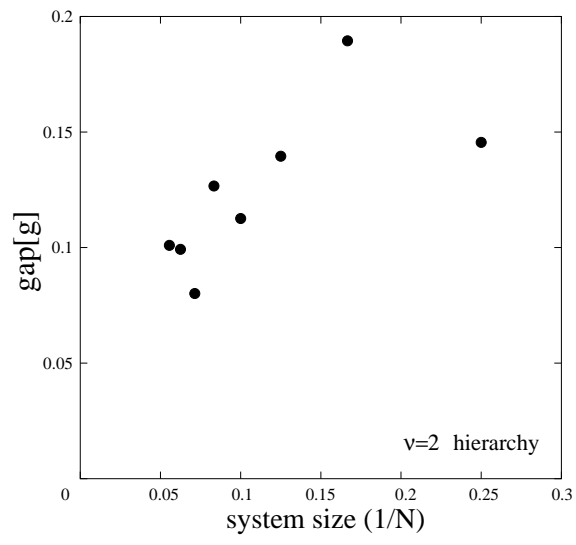

Figure 7: The gaps of the candidate states for a hierarchical $\nu=2$ fraction.

This is consistent with the proposal that in this regime of filling factors the system has ground states belonging to a family of states containing clusters of $k$ particles, the Read-Rezayi states [14, 39. The corresponding wavefunctions are constructed by symmetrizing products of $k$ Laughlin states. For $k=1$ this gives simply the Laughlin state Eq.(6) and for $k=2$ the Pfaffian state Eq.(15). The filling factor is given by $\nu=k / 2$ and on the sphere they require the special flux $2 S=(2 / k) N-2$. For $\nu=3 / 2$ we are able to study $\mathrm{N}=6,9,12,15$ and for $\nu=2 \mathrm{~N}=8,12,16$. Again the spectra shows the features associated with FQHE states. We have obtained the neutral gap of these states. However it 
is difficult to draw any firm conclusion concerning the limit of large system size because the gaps are badly behaved : see Figs.(8a,b).
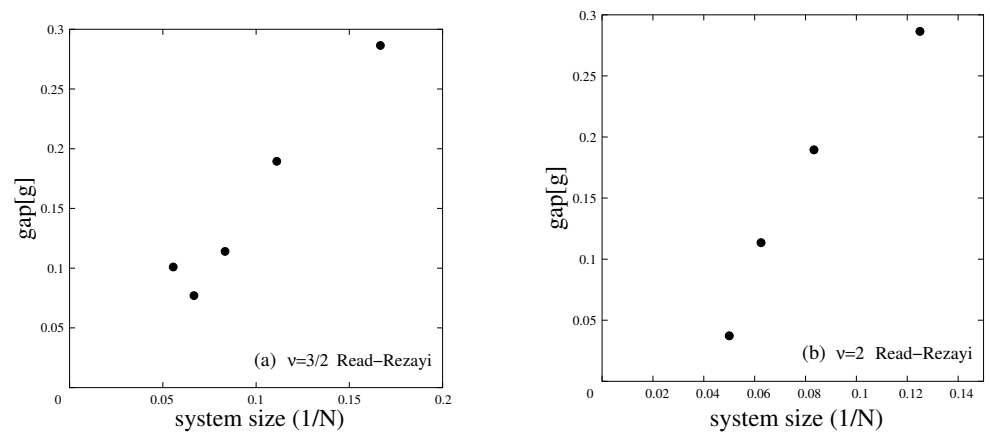

Figure 8: Gaps for the possible Read-Rezayi states on the sphere : in (a) the fraction $\nu=3 / 2$ is displayed (b) the fraction $\nu=2$.

Finally we note that the Read-Rezayi states have several quasihole states : adding one flux quantum to the fiducial state leads to the production of $k$ quasiholes. They should appear as a set of low-lying levels with an easily computable degeneracy. This degeneracy would be exact for the model Hamiltonian with $(k+1)$-body forces of Read and Rezayi. For the system sizes of this work, this does not appear clearly but is not surprising. It is likely that these fractions have large correlation lengths and the finite size effects are still too large.

\section{CONCLUSIONS}

In this paper we have studied the occurrence of quantum Hall fractions of spinless bosons in a rotating trap in the lowest Landau level. We have used exact diagonalization techniques in the spherical geometry. We gave evidence for the appearance of the bosonic analog of Jain principal sequence of FQHE fractions $\nu=p /(p \pm 1)$ instead of $\nu=p /(2 p \pm 1)$ for fermions. This is exactly what is expected from standard arguments based on Fermi-Bose correspondence and Chern-Simons theories, albeit not rigorously established. In the spectra of these fractions, we have shown that there exists states corresponding to the expected collective density mode of the FQHE states. The angular momentum extent of the collective mode is in agreement with the standard physics of FQHE fractions. There is so far no evidence for fractions outside the principal sequence. This is not so if we modify the potential between bosons : then notably the fraction $\nu=2 / 5$ appears clearly. This principal sequence cannot describe the physics of the bosonic fluid for fillings larger than one. In fact its two members $\nu=2$ as well as $\nu=3 / 2$ do not show any indication of convergence towards the thermodynamic limit.

For complete filling of the lowest Landau level, we have shown that the potential Fermi sea of composite fermions is destroyed and that a paired state, the Pfaffian state is likely to be the ground state. There is a series of incompressible states that has exactly the shift predicted by the Pfaffian wavefunction. The gap extrapolates smoothly to a nonzero value and in addition there is good evidence that half-flux excitations typical of this paired state are present. At the points in the $(\mathrm{N}, 2 \mathrm{~S})$ plane predicted by Read and Rezayi we find also FQHE states for fillings larger than one. In this case their ultimate fate in the thermodynamic limit is not clearly established from the system sizes we have been able to treat. This remains a subject for future studies.

\section{Acknowledgments}

We thank Jean Dalibard for fruitful discussions. The numerical calculations have been performed thanks to a computer time allocation of IDRIS-CNRS.

[1] R. B. Laughlin, Phys. Rev. Lett. 50, 1395 (1983).

[2] F. D. M. Haldane, Phys. Rev. Lett. 51, 605 (1983).

[3] M. R. Matthews, B. P. Anderson, P. C. Haljan, D. S. Hall, C. E. Wieman, and E. A. Cornell, Phys. Rev. Lett. 83, 2498 (1999). 
[4] K. W. Madison, F. Chevy, W. Wohlleben, and J. Dalibard, Phys. Rev. Lett. 84, 806 (2000); F. Chevy, K. Madison, and J. Dalibard, Phys. Rev. Lett. 85, 2223 (2000).

[5] P. C. Haljan, I. Coddington, P. Engels, and E. A. Cornell, Phys. Rev. Lett. 87, 210403 (2001).

[6] J. R. Abo-Shaeer, C. Raman, J. M. Vogels , and W. Ketterle, Science 292, 476 (2001).

[7] P. Rosenbusch, D. S. Petrov, S. Sinha, F. Chevy, V. Bretin, Y. Castin, G. Shlyapnikov, and J. Dalibard, Phys. Rev. Lett. 88, 250403 (2002).

[8] N. K. Wilkin, J. M. F. Gunn, and R. A. Smith, Phys. Rev. Lett. 80, 2265 (1998).

[9] N. R. Cooper and N. K. Wilkin, Phys. Rev. B60, R16279 (1999).

[10] N. K. Wilkin and J. M. F. Gunn, Phys. Rev. Lett. 84, 6 (2000).

[11] N. R. Cooper, N. K. Wilkin, and J. M. F. Gunn, Phys. Rev. Lett. 87, 120405 (2001).

[12] B. Paredes, P. Fedichev, J. I. Cirac, and P. Zoller, Phys. Rev. Lett.87, 010402 (2001); B. Paredes, P. Zoller, and J. I. Cirac, Phys. Rev. A 66, 033609 (2002).

[13] G. Moore and N. Read, Nucl. Phys. B360, 362 (1991).

[14] N. Read and E. H. Rezayi, Phys. Rev. B54, 16864 (1996); Phys. Rev. B59, 8084 (1999).

[15] J. W. Reijnders, F. J. M. van Lankvelt, K. Schoutens, and N. Read, Phys. Rev. Lett. 89120401 (2002).

[16] J. W. Reijnders, F. J. M. van Lankvelt, K. Schoutens, and N. Read, "Rotating spin-1 bosons in the lowest Landau level", e-print cond-mat/0306402.

[17] N. R. Cooper, "Exact Groundstates of Rotating Bose Gases close to a Feshbach Resonance", e-print cond-mat/0308283.

[18] N. Regnault and Th. Jolicoeur, Phys. Rev. Lett. 91030402 (2003).

[19] F. D. M. Haldane and E. H. Rezayi, Phys. Rev. Lett. 54, 237 (1985).

[20] M. A. Cazalilla, Phys. Rev. A67, 063613 (2003).

[21] S. Trugman and S. Kivelson, Phys. Rev. B59, 8084 (1985).

[22] S. Viefers, T. H. Hansson, and S. M. Reimann, Phys. Rev. A62, 053604 (2000).

[23] G. F. Bertsch and T. Papenbrock, Phys. Rev. Lett. 83, 5412 (1999).

[24] I. Tamm, Z. Phys. 71, 141 (1931).

[25] T. T. Wu and C. N. Yang, Nucl. Phys. B107, 365 (1976); Phys. Rev. D16, 1018 (1977).

[26] G. Fano, F. Ortolani, and E. Colombo, Phys. Rev. B34, 2670 (1986).

[27] S. M. Girvin, A. H. MacDonald, and P. M. Platzman, Phys. Rev. Lett. 54, 581 (1985); Phys. Rev. B33, 2481 (1996).

[28] J. K. Jain, Phys. Rev. Lett. 63, 199 (1989); Phys. Rev. B41, 7653 (1990).

[29] O. Heinonen (Ed.), "Composite Fermions", World Scientific, New York, 1998.

[30] N. d'Ambrumenil and R. Morf, Phys. Rev. B40, 6108 (1989).

[31] B. I. Halperin, P. A. Lee, and N. Read, Phys. Rev. B47, 7312 (1993).

[32] E. H. Rezayi and N. Read, Phys. Rev. Lett. 72, 900 (1994).

[33] M. Greiter, X. G. Wen, and F. Wilczek, Phys. Rev. Lett. 66, 3205 (1991); Nucl. Phys. B374, 567 (1992); Phys. Rev. B46, 9586 (1992).

[34] M. Milovanovic and N. Read, Phys . Rev. B53, 13559 (1996).

[35] There is a presentation of the BCS Pfaffian in P. G. de Gennes, "Superconductivity of metals and alloys", Benjamin, New York (1966), p. 106-107.

[36] R. Morf, Phys. Rev. Lett. 80, 1505 (1998).

[37] E. H. Rezayi and F. D. M. Haldane, Phys. Rev. Lett. 84, 4685 (2000).

[38] see the note at ref.30 in G. S. Canright and S. M. Girvin, Int. J. Mod. Phys. B3, 1943 (1989).

[39] N. Read and D. Green, Phys . Rev. B61, 10267 (2000).

[40] X. C. Xie, S. He, and S. Das Sarma, Phys. Rev. Lett. 66, 389 (1991).

[41] J. Sinova, C. B. Hanna, and A. H. MacDonald, Phys. Rev. Lett. 89, 030403 (2002). 